\title{
Barrett-Ösophagus: Malignomrisiko geringer als erwartet
}

\author{
Dass der Barrett-Ösophagus eine Präkanzerose \\ für das distale Ösophaguskarzinom darstellt, gilt \\ heute als gesichert. Doch wie hoch ist das Risiko, \\ dass ein betroffener Patient in den nächsten \\ zehn Jahren ein solches Karzinom entwickelt?
}

- Um diese Frage zu beantworten, wurde der Krankheitsverlauf von 42207 Patienten mit einem Barrett-

Ösophagus analysiert. Bei 8\% dieser Patienten lag bereits eine niedriggradige Dysplasie vor. Die Auswertung der Daten ergab, dass das jährliche Risiko, ein ösophageales Adenokarzinom zu entwickeln, nur bei $0,4 \%$ liegt, d.h. innerhalb von zehn Jahren erkranken 4\% der Patienten mit einer Barrett-Schleimhaut an diesem Tumor. Männliches Geschlecht, fortgeschrittenes Alter und niedriggradi-

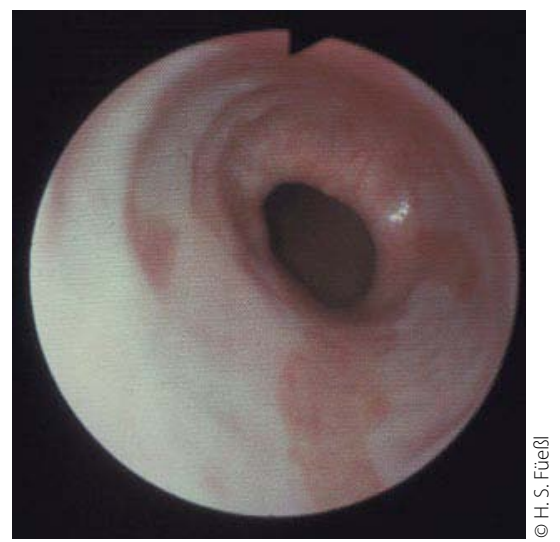

Der Barrett-Ösophagus gilt als Präkanzerose. ge Dysplasien erwiesen sich als unabhängige Prädiktoren für eine maligne Entartung.

\section{Kommentar}

Auch wenn der Barrett-Ösophagus eine Präkanzerose darstellt, ist die Effektivität einer intensiven Überwachungsstrategie abhängig von der Inzidenzrate. Die vorliegenden Daten zeigen, dass das Risiko bei fehlender bzw. niedriggradiger Dysplasie doch relativ gering ist. Ob sich daraus neue Empfehlungen für die Kontrollintervalle ableiten lassen, darüber wird man sicherlich diskutieren müssen.

P. Stiefelhagen!

- P.J.F. de Jonge et al.

Risk of malignant progression in patients with Barrett's esophagus: A Dutch nationwide cohort study. Gut 59 (2010) 1030-1036 